\title{
Cryoballoon Ablation Strategy in Persistent Atrial Fibrillation
}

\author{
Florian Straube ${ }^{1,2 * t}$, Janis Pongratz ${ }^{1 \dagger}$, Alexander Kosmalla ${ }^{1}$, Benedikt Brueck ${ }^{1,3}$, \\ Lukas Riess ${ }^{1}$, Stefan Hart ${ }^{1,4}$, Christian Tesche ${ }^{1,2,5}$, Ullrich Ebersberger ${ }^{1,2,6}$, \\ Michael Wankerl ${ }^{1}$, Uwe Dorwarth ${ }^{1}$ and Ellen Hoffmann ${ }^{1}$
}

\begin{abstract}
${ }^{1}$ Department of Cardiology and Internal Intensive Care Medicine, Heart Center Munich-Bogenhausen, Munich Clinic Bogenhausen, Academic Teaching Hospital of the Technical University Munich, Munich, Germany, ${ }^{2}$ Faculty Munich University Clinic, Ludwig-Maximilians-University, Munich, Germany, ${ }^{3}$ KardiologieErkelenz, Erkelenz, Germany, ${ }^{4}$ Cardiology, University of Düsseldorf, Düsseldorf, Germany, ${ }^{5}$ Department of Cardiology, Klinik Augustinum, Munich, Germany,

${ }^{6} \mathrm{KMN}-$ Kardiologie Muenchen Nord, Munich, Germany
\end{abstract}

\section{OPEN ACCESS}

Edited by:

Richard Hauer,

University Medical Center

Utrecht, Netherlands

Reviewed by:

Hugh Calkins,

Johns Hopkins Medicine,

United States

Giannis G. Baltogiannis,

Vrije University Brussel, Belgium

*Correspondence:

Florian Straube

florian.straube@muenchen-Klinik.de orcid.org/0000-0002-4491-5224

tThese authors have contributed equally to this work and share first authorship

Specialty section: This article was submitted to Cardiac Rhythmology, a section of the journa Frontiers in Cardiovascular Medicine

Received: 13 August 2021 Accepted: 07 October 2021 Published: 18 November 2021

Citation:

Straube F, Pongratz J, Kosmalla A, Brueck B, Riess L, Hartl S, Tesche C,

Ebersberger U, Wankerl $M$,

Dorwarth U and Hoffmann E (2021) Cryoballoon Ablation Strategy in Persistent Atrial Fibrillation.

Front. Cardiovasc. Med. 8:758408

doi: 10.3389/fcvm.2021.758408
Background: Cryoballoon ablation is established for pulmonary vein isolation (PVI) in paroxysmal atrial fibrillation (AF). The objective was to evaluate CBA strategy in consecutive patients with persistent AF in the initial AF ablation procedure.

Material and Methods: Prospectively, patients with symptomatic persistent AF scheduled for AF ablation all underwent cryoballoon PVI. Technical enhancements, laboratory management, safety, single-procedure outcome, predictors of recurrence, and durability of PVI were evaluated.

Results: From 2007 to 2020, a total of 1,140 patients with persistent AF, median age 68 years, underwent cryoballoon ablation (CBA). Median left atrial (LA) diameter was $45 \mathrm{~mm}$ (interquantile range, IQR, 8), and Congestive heart failure, Hypertension, Age $\geq 75$ years (doubled), Diabetes mellitus, prior Stroke or TIA or thromboembolism (doubled), Vascular disease, Age 65 to 74 years, Sex category $\left(\mathrm{CHA}_{2} \mathrm{DS}_{2}\right.$-VASc) score was 3. Acute isolation was achieved in $99.6 \%$ of the pulmonary veins by CBA. Median LA time and median dose area product decreased significantly over time $(p<0.001)$. Major complications occurred in 17 (1.5\%) patients including $2(0.2 \%)$ stroke/transitory ischemic attack (TIA), 1 (0.1\%) tamponade, relevant groin complications, 1 (0.1\%) significant ASD, and 4 (0.4\%) persistent phrenic nerve palsy (PNP). Transient PNP occurred in 66 (5.5\%) patients. No atrio-esophageal fistula was documented. Five deaths $(0.4 \%)$, unrelated to the procedure, occurred very late during follow-up. After initial CBA, arrhythmia recurrences occurred in $46.6 \%$ of the patients. Freedom from atrial arrhythmias at 1-, and 2-year was 81.8 and $61.7 \%$, respectively. Independent predictors of recurrence were LA diameter, female sex, and use of the first cryoballoon generation. Repeat ablations due to recurrences were performed in 268 (23.5\%) of the 1,140 patients. No pulmonary vein $(\mathrm{PV}$ ) reconduction was found in $49.6 \%$ of the patients and $73.5 \%$ of $\mathrm{PV}$ s. This rate increased to $66.4 \%$ of the patients and $88 \%$ of PVs if an advanced cryoballoon was used in the first AF ablation procedure.

Conclusion: Cryoballoon ablation for symptomatic persistent AF is a reasonable strategy in the initial AF ablation procedure.

Keywords: atrial fibrillation, catheter ablation, cryoballoon ablation, pulmonary vein isolation, persistent atrial fibrillation 


\section{INTRODUCTION}

In symptomatic persistent atrial fibrillation (AF), catheter ablation is an established treatment option (1). Pulmonary vein isolation (PVI) is the cornerstone of $\mathrm{AF}$ ablation and is recommended with a class I, level of evidence $\mathrm{A}$ in the current guideline (2). Additional ablation procedures beyond PVI are not well-established; therefore, current guidelines give a class IIb recommendation $(1,2)$.

Cryoballoon ablation is a standard treatment procedure for symptomatic paroxysmal AF (1) and has become a firstline treatment for this type of symptomatic AF recently (35). Cryoballoon ablation (CBA) for symptomatic persistent $\mathrm{AF}$ has been evaluated in a pilot study with the less effective first generation cryoballoon (CBG1): A double balloon strategy with the concomitant treatment with both, the 23 and $28 \mathrm{~mm}$ cryoballoon aiming at broad and wide circumferential antral ablation demonstrated favorable initial results (6). After the introduction of the more effective second-generation cryoballoon (CBG2) in 2012, the single big balloon strategy became an option for PVI in persistent AF.

A CBA system (Arctic Front ${ }^{\mathrm{TM}}$; Cryocath Inc.) was approved by the European Union (EU) for the interventional treatment of AF in 2005, and the Food and Drug Administration (FDA) approved the system in 2010 (Arctic Front ${ }^{\mathrm{TM}}$, Medtronic Inc.) to treat drug-refractory paroxysmal AF. Recently, in June 2020, the FDA extended the labeling for the treatment of drug-refractory recurrent symptomatic persistent AF (episode duration $<6$ months) mainly based on the results of the CRYO4PERSISTENT single-arm trial (7): A total of 101 patients with early persistent AF were treated by means of CBA. Single-procedure success was $61 \%$ at 12 months post-ablation in addition to significant reduction in arrhythmia-related symptoms and improved quality of life (7).

In 2018, Omran et al. included 917 patients with persistent AF from 11 CBA studies into a meta-analysis. Freedom from AF was $68.9 \%$ at 16.7 months (8). Most of the studies were observational, and selected patients were included. The number of patients was limited in all of the studies ( $\leq 180$ patients).

The objective of this large all-comer study was to evaluate and share our experience with $\mathrm{CBA}$ as the exclusive ablation strategy in consecutive patients for the initial ablation procedure in symptomatic persistent AF. Efficacy, safety, and information on the durability of PVI from repeat procedures were evaluated.

\section{MATERIALS AND METHODS}

Consecutive patients with symptomatic persistent AF scheduled for the first $\mathrm{AF}$ ablation procedure were prospectively included into the observational single-center study. All the patients were treated by means of CBA. Exclusion criteria were according to the guidelines. Patients with LA diameter $>60 \mathrm{~mm}$ were not referred for AF ablation. Patients with long-standing persistent AF were pretreated with antiarrhythmic drugs, and sinus rhythm was established by cardioversion. Cessation of anti-arrhythmic drug $(\mathrm{AAD})$ treatment was recommended after the procedure.
Procedural and periprocedural results, complications, and the outcome were documented. The primary endpoint was freedom from any atrial arrhythmia recurrences, AF or atrial tachycardia (AT), and off antiarrhythmic drugs following a 90-day blanking period. Patients with $\mathrm{AAD}$ at follow-up were calculated as failures after the blanking period. Relevant typical AF symptoms $>30 \mathrm{~s}$ reported by the patients without any ECG documentation were calculated as a recurrence.

\section{Cryoballoon Procedure}

The procedure has been described in detail in previous studies $(6,9-14)$. Prior to the procedure, baseline characteristics were documented, and all the patients underwent transthoracic and transesophageal echocardiography.

The procedure was performed under conscious sedation. After venous access, single transseptal puncture was performed with a guiding sheath and BRK needle and was guided by transesophageal or intracardiac echocardiography. A steerable sheath (Flexcath or Flexcath Advance ${ }^{\mathrm{TM}}$; Medtronic Inc., Minneapolis, MN, United States) was positioned in the left atrium over a guidewire. Four different cryoballoon generations were used depending on its availability in the study: Arctic Front $^{\mathrm{TM}}$ (CBG1), Arctic Front Advance ${ }^{\mathrm{TM}}$ (CBG2), Arctic Front Advance $\mathrm{ST}^{\mathrm{TM}}$ (CBG3), and Arctic Front Advance Pro ${ }^{\mathrm{TM}}$ (CBG4), which were all from Medtronic Inc. (Minneapolis, MN, United States). The cryoballoon was introduced over a stiff wire or the spiral mapping catheter (SMC) and positioned at the ostium of the PV. Three different SMCs with different sizes were available during the inclusion period: a 6-pole mapping catheter with a 15-mm loop (ProMap ${ }^{\text {TM}}$; ProRhythm Inc., Ronkonkoma, NY, United States), or an 8-pole with 15- or 20-mm loop (Achieve $^{\mathrm{TM}}$ or Achieve Advance ${ }^{\mathrm{TM}}$ ), or a 10 -pole with $25-\mathrm{mm}$ loop (Achieve Advance ${ }^{\mathrm{TM}}$ ) (the latter are all from Medtronic Inc., Minneapolis, MN, United States). ACT was kept between 300 and $400 \mathrm{~s}$ with unfractionated heparin. PV potentials were recorded at least before and after complete PVI with a circular mapping catheter. Periprocedural management was performed in accordance with the current practice guidelines (2). Balloon positioning was visualized by fluoroscopy and intracardiac echocardiography if available. Immediately before the start of the cryoablation cycle, occlusion was determined with a contrast injection through the inner lumen of the cryoballoon.

With the introduction of the CBG1, with an equatorial cooling zone, we applied a double balloon strategy as part of a persistent AF proof of concept study using both the 23- and 28$\mathrm{mm}$ balloons aiming at optimized PV occlusion and isolation with the smaller balloon followed by a wide circumferential lesion induction with the larger balloon. With the availability of the more effective CBG2 (broader cooling zone of the distal hemisphere of the balloon, increased refrigerant flow rate), the strategy was adapted to the primary use of the $28-\mathrm{mm}$ balloon as the catheter. In case of inability to isolate the PV with the described setting, it was at the discretion of the operator to change the SMC to a stiff wire, use the small $23 \mathrm{~mm}$ cryoballoon only in PVs with an ostial maximum diameter of $\leq 21 \mathrm{~mm}$, or use RF touch-up applications to isolate the PV. The institutional 
$\begin{array}{llllllllllllll}\text { Timeline } & 2007 / 8 & 2009 & 2010 & 2011 & 2012 & 2013 & 2014 & 2015 & 2016 & 2017 & 2018 & 2019 & 2020\end{array}$

\section{Technical Enhancements}

\begin{tabular}{|c|c|c|c|c|c|c|}
\hline CB generations & CBG1 & & CBG2 & CBG3 & CBG2 & CBG4 \\
\hline Spiral mapping & $\begin{array}{c}15 \mathrm{~mm} \\
6 \text { electrodes }\end{array}$ & $\begin{array}{l}\mathrm{G} 1: 15 \mathrm{~mm} \\
8 \text { electrodes }\end{array}$ & $\begin{array}{l}\mathrm{G} 2: 20 \mathrm{~mm} \\
8 \text { electrodes }\end{array}$ & \multicolumn{3}{|c|}{$\begin{array}{c}\mathrm{G} 2: 20 \mathrm{~mm}(25 \mathrm{~mm}) \\
8(10) \text { electrodes }\end{array}$} \\
\hline
\end{tabular}

\begin{tabular}{l|l} 
Steerable Sheath & first-generation CB sheath \\
\cline { 2 - 3 }
\end{tabular}

\section{EP Lab Management}

\begin{tabular}{|c|c|c|c|}
\hline \multirow{2}{*}{$\begin{array}{l}\text { Analgosedation } \\
\text { Anticoagulation }\end{array}$} & \multicolumn{2}{|l|}{ Diazepam/Fentanyl } & ropofol/Morphine \\
\hline & interrupted OAC & \multicolumn{2}{|c|}{ short or uninterrupted OAC } \\
\hline Access site & Sheath removal at IMC ward & \multicolumn{2}{|c|}{ Protamine, sheath removal at EP lab } \\
\hline ET measurement & \multicolumn{2}{|c|}{ Longitudinal ET probe w. 3 temp. electrodes } & S-shape ETP, 12 ele \\
\hline
\end{tabular}

\section{Ablation Procedure}

\begin{tabular}{l|c|c|c|}
\cline { 2 - 4 } Strategy & \multicolumn{2}{c|}{ Double balloon strategy } & \multicolumn{2}{c|}{$28 \mathrm{~mm}$ balloon } \\
\cline { 2 - 4 } Imaging & Fluoroscopy + Cine + TEE & \multicolumn{2}{|c|}{ Fluoroscopy + Cine + ICE } \\
\cline { 2 - 4 } Ablation protocol & $2 \times 300 \mathrm{~s}$ & $\begin{array}{l}2 \times 240 \mathrm{~s} \\
2 \times 180 \mathrm{~s}\end{array}$ & TTI guided $\leq 45 \mathrm{~s} \rightarrow 180 \mathrm{~s}$ no bonus \\
\cline { 2 - 4 } & &
\end{tabular}

FIGURE 1 | Evolution of cryoballoon ablation for persistent atrial fibrillation (AF) from 2007 to 2020. A timeline of technical enhancements, improvement in laboratory management, and implementation of new cryoballoon protocols are shown in the upper part of the figure. CB, cryoballoon; CBG, cryoballoon generation; EP, electrophysiology; ET, esophageal temperature; ICE, intracardiac echocardiography; TEE, transesophageal echocardiography; TTI, time-to-isolation.

protocol for all cryoballoon ablation procedures evolved from 2007 to 2020 (Figure 1).

An endoluminal esophageal linear (SensiTherm; St. Jude Medical, Saint Paul, MN, United States) or sinusoidal temperature probe with 12 thermocouples (CIRCA S-Cath ${ }^{\mathrm{TM}}$; CIRCA Scientific, Englewood, CO, United States) was used in all the patients. Phrenic nerve (PN) stimulation was controlled by palpation and intracardiac echocardiography visualization of the diaphragmatic motion if available. For premature termination due to esophageal temperatures $\leq+15^{\circ} \mathrm{C}$, $\mathrm{PN}$ impairment or balloon temperatures $\leq-55^{\circ} \mathrm{C}$ for the right superior $\mathrm{PV}$, double stop technique was applied after its introduction in 2012/2013. See details for the evolution of the modification of the ablation protocol in Figure 1.

\section{Post-procedure Care}

Transthoracic echocardiography was performed in all the patients to rule out pericardial effusion. From 2007 to 2013, sheath removal was performed in the intermediate care ward after activated clotting time decreased to $\leq 200$ s. Since 2013 , protamine has been administered immediately before sheath removal in the lab. From 2007 to 2013, vitamin K antagonists (VKAs) were interrupted up to 7 days before the intervention, and bridging with low-molecular weight heparin was performed. Intravenous heparin was used after the procedure until the next day. Low molecular-weight heparin was administered to patients on VKAs with INR $<2$ until therapeutic INR 2-3 was achieved.
Since 2013, in patients on VKAs with an INR of 2 to 3, the procedure has been carried out without discontinuation of the oral anticoagulation. In patients on novel oral anticoagulants, the intake of a novel oral anticoagulant in the morning was postponed until after the procedure. Novel oral anticoagulants were reinitiated after hemostasis and no later than $1 \mathrm{~h}$ after ablation. Anticoagulation was continued for $\geq 3$ months, and thereafter based on individual $\mathrm{CHA}_{2} \mathrm{DS}_{2}$-VASc score. All the patients were empirically treated with proton-pump inhibitors for 4 weeks.

\section{Study Follow-Up}

All the patients were monitored during the hospital stay for at least $24-48 \mathrm{~h}$ post intervention, including continuous monitoring in the intermediate care ward, by telemetry and/or 24-72-h Holter studies.

Routine follow-up included symptom evaluation, 12-lead ECG, and Holter studies (1-7 days). Follow-up was performed by the outpatient clinic in collaboration with referring physicians and by structured telephone interview. In patients with repeat ablation following CBA due to recurrence, data on the durability of PVI were documented prospectively.

\section{Statistical Analysis}

In accordance with the Shapiro-Wilk test, continuous variables are expressed as means with standard deviation (SD) or as medians with quartiles. Categorical data are shown as 
TABLE 1 | Baseline characteristics and differences in the treatment groups.

\begin{tabular}{|c|c|c|c|c|c|c|}
\hline & Total & CBG1 & CBG2 & CBG3 & CBG4 & $p$-value \\
\hline N patients (\%) & $1,140(100)$ & $208(18.2)$ & $613(53.8)$ & $112(9.8)$ & $207(18.2)$ & \\
\hline Age, years & 68 [15] & 63 [13] & 69 [15] & $69[17]$ & 69 [16] & $<0.001$ \\
\hline Females (\%) & 391 (34.3) & 49 (23.6) & $232(35.7)$ & $40(35.7)$ & $70(33.8)$ & 0.003 \\
\hline LA diameter ${ }^{\star} \mathrm{mm}$ & $45[8]$ & $44[5]$ & $45[8]$ & $44[6]$ & $44[8]$ & 0.001 \\
\hline Ejection fraction\% & $55[10]$ & $60[8]$ & $55[10]$ & $55[5]$ & $55(9)$ & $<0.001$ \\
\hline AAD I/III prior to the procedure (\%) & $310 / 465$ (66.7) & 15/22 (68.2) & 170/242 (70.2) & 19/28 (67.9) & 106/173 (61.3) & 0.30 \\
\hline Number of electrical cardioversions & $2[2] n=521$ & $\mathrm{n} / \mathrm{a}$ & $2[2] n=283$ & $2[2] n=30$ & $2[2] n=207$ & 0.09 \\
\hline Number of Episodes per year & $3[3] n=470$ & $\mathrm{n} / \mathrm{a}$ & $3[4] n=254$ & $3[3] n=30$ & $3[3] n=185$ & 0.97 \\
\hline Max. duration of a single AF episode, days & $30[80] n=460$ & $\mathrm{n} / \mathrm{a}$ & $30[80] n=258$ & $7[21] n=29$ & $30[75] n=172$ & 0.001 \\
\hline Hypertension & 819 (73.3) & $130(62.5)$ & $455(76.1)$ & $78(75.0)$ & $156(75.4)$ & 0.002 \\
\hline Hypertensive heart disease & $403(36.1)$ & 38 (18.3) & $225(37.6)$ & $50(48.1)$ & $90(43.7)$ & $<0.001$ \\
\hline Mitral regurgitation $\geq \|$ & $61(5.4)$ & $6(2.9)$ & $37(6.0)$ & $4(3.6)$ & $14(6.8)$ & 0.20 \\
\hline Coronary artery disease & $283(24.8)$ & $32(15.4)$ & $149(24.3)$ & $28(25.0)$ & $74(35.7)$ & $<0.001$ \\
\hline Prior myocardial infarction & $36(3.2)$ & $3(1.4)$ & 19 (3.2) & $4(3.8)$ & $10(4.9)$ & 0.26 \\
\hline Cardiomyopathy & $148(13.0)$ & $13(6.3)$ & $78(12.7)$ & $19(17.0)$ & $38(18.4)$ & 0.002 \\
\hline Structural heart disease & $334(29.7)$ & $86(41.3)$ & 175 (19.2) & $62(55.4)$ & $11(5.4)$ & $<0.001$ \\
\hline Diabetes mellitus & $41 / 543(7.6)$ & 3/24 (12.5) & 23/284 (8.1) & 1/30 (3.3) & 14/205 (6.8) & 0.60 \\
\hline $\mathrm{CHA}_{2} \mathrm{DS}_{2}$-VASc Score & $3[2] n=486$ & $\mathrm{n} / \mathrm{a}$ & $3[2] n=263$ & $3[2] n=28$ & $3[2] n=194$ & 0.61 \\
\hline $\mathrm{BMl} \mathrm{kg/m^{2 }}$ & $27.5[6.1]$ & $28.6[7.8]$ & $27.1[5.9]$ & $26.4[4.2]$ & $26.6[6.5]$ & 0.008 \\
\hline Overweight (BMI > 25) & $593(52.5)$ & $63(30.3)$ & $350(57.1)$ & $49(43.8)$ & $131(63.3)$ & $<0.001$ \\
\hline Obesity (BMI > 30) & $242(21.2)$ & $30(14.4)$ & $140(22.8)$ & $16(14.3)$ & $56(27.1)$ & 0.003 \\
\hline Obesity ㅇl/III (BMI > 35) & 75 (6.6) & $14(6.7)$ & $42(6.9)$ & $3(2.7)$ & $16(7.7)$ & 0.35 \\
\hline Common ostium & $88(7.7)$ & $11(5.3)$ & $56(9.1)$ & $11(9.8)$ & $10(4.8)$ & 0.09 \\
\hline Accessory veins & $51(4.5)$ & $17(8.2)$ & $29(4.7)$ & $4(3.6)$ & $1(0.5)$ & 0.002 \\
\hline
\end{tabular}

$N(\%)$ : number of patients and percentage; mean \pm standard deviation or median with interquartile range [in squared brackets] as appropriate according to the test of normal distribution.

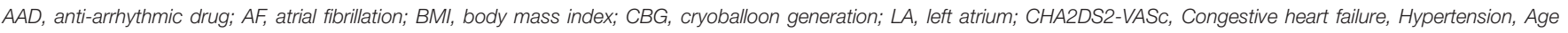

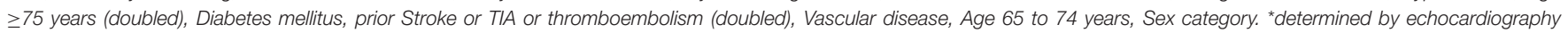
(anterior-posterior diameter).

Bold values are referred statistically significant.

numbers and percentages and are compared by the chi-square test. Friedman's two-way analysis of variance by Ranks was performed to test for differences between groups, if more than two groups with non-normal distribution were compared. Kaplan-Meier Survival Curve for the endpoint freedom from any atrial arrhythmia recurrence was calculated, and logrank test was performed for comparison. Univariate Cox regression analyses were performed to identify predictors of recurrence. Main independent risk factors were determined using a stepwise multivariate Cox regression model with bidirectional elimination including only parameters of highest univariate significance. Database management system FileMaker Pro (Claris International Inc., Santa Clara, CA, United States) was used. Data processing and analysis were performed using Excel 2010 (Microsoft Corp., Redmond, WA, United States) and SPSS 20.0 (IBM Corp., Armonk, NY, United States). Statistical significance was defined as $p \leq 0.05$.

\section{RESULTS}

\section{Study Population and Differences Among the Treatment Groups}

From 2007 to 2020, 1,140 consecutive patients with symptomatic persistent atrial fibrillation underwent AF ablation at the Heart
Center Munich-Bogenhausen. All of them underwent electrical disconnection of the pulmonary veins by means of CBA. The procedures were performed by five experienced operators. Four different cryoballoon generations were available over time (Figure 1), and treatment groups were created accordingly. Baseline characteristics were different in the treatment groups. Older age, more females, larger LA diameter sizes, lower ejection fraction, more hypertension and hypertensive heart disease, more coronary heart disease and cardiomyopathies were observed in the treatment groups with the advanced cryoballoon catheters (CBG2, CBG3, and CBG4) as compared with CBG1. A significant decline of the body mass index was observed over time (see Table 1 for details). No significant difference was observed for the finding of the left common ostium, but significantly more right accessory PVs were documented with the use of CBG1.

\section{Advances in CBA for Persistent AF}

The introduction of the second cryoballoon generation in 2012 with an increased refrigerant flow rate and a broader cooling zone led to a continuous adaption of the ablation protocol. The reduction of freezing time per application, implementation of time-to-isolation guided ablation protocols, omission of the double balloon strategy, and omission of the routine bonus application became possible. In addition to the learning curve of the operators over more than a decade, lab 


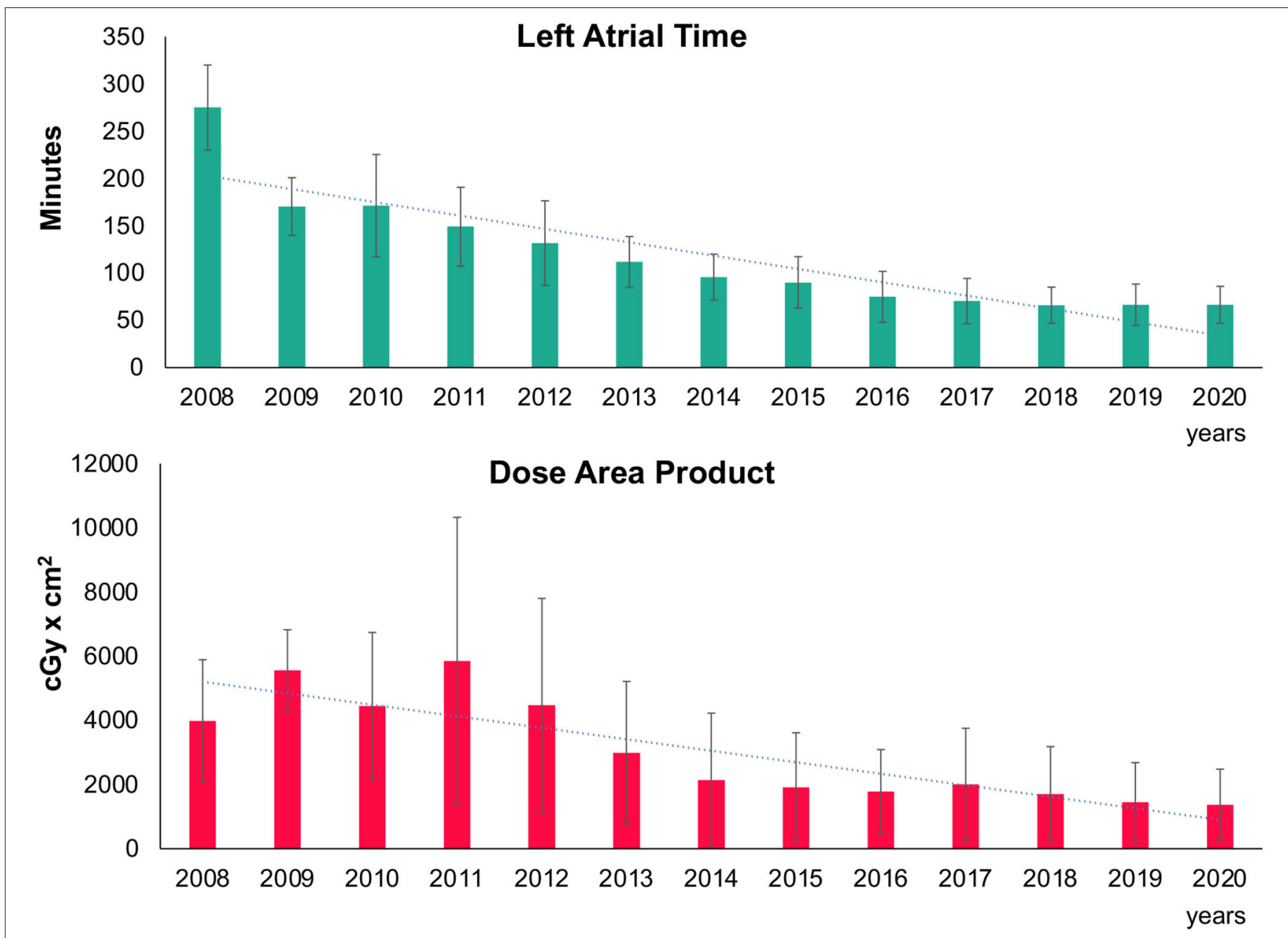

FIGURE 2 | Trend in left atrial procedure time and radiation. Mean left atrial procedure time (upper part) and dose area product (lower part) with standard deviation are depicted in bar charts over time. Trend line shows the decline over the years.

management was optimized by the implementation of modern analgo-sedation, optimized access site management, advanced esophageal temperature monitoring, and use of intracardiac echocardiography. Details are presented in Figure 1.

\section{Acute Efficacy and Effectiveness}

The acute efficacy measured by the rate of isolated PV at the end of the procedure increased within the treatment groups ranged from 99.1 to $100 \%, p=0.011$. After the introduction of the advanced cryoballoons (Arctic Front Advance ${ }^{\mathrm{TM}} /$ Advance $\mathrm{ST}^{\mathrm{TM}} /$ Advance Pro ${ }^{\mathrm{TM}}$; all from Medtronic Inc.), the double balloon rate decreased significantly $(p<0.001)$. A continuous and statistically significant decrease in LA dwelling time (Figure 2), fluoroscopy time, and dose area product (Figure 2), which represents the radiation risk from the interventional procedure, were found (see Table 2 for details).

\section{Safety}

The rate of adverse events following the procedure is presented in Table 3 for the total population and treatment groups.
No procedure-related death and no atrioesophageal fistula were observed. Five deaths $(0.4 \%)$ unrelated to the procedure occurred very late during long-term follow-up. The major complication rate was $1.5 \%$ in the total population, and no difference was observed among the treatment groups. Pericardial tamponade was observed in 1 of the 1,140 procedures $(0.09 \%)$. The rate of thromboembolic cerebrovascular complications was $0.2 \%$. Minor adverse events were observed in $8.9 \%$ of the patients, and included $5.3 \%$ transient phrenic nerve palsy (PNP), which recovered completely until discharge of the patients. The rate of transient PNP decreased significantly over time. The rate of persistent phrenic nerve palsy was $0.4 \%$. Pulmonary infections were infrequent after the CBA, and the rate declined significantly among the treatment groups.

The change of the analgo-sedation regime from intermittent diazepam and fentanyl application to continuous propofol and intermittent morphine application, and the implementation of uninterrupted or shortly interrupted oral anticoagulation were both not associated with more complications. 
TABLE 2 | Procedural characteristics and differences in the treatment groups.

\begin{tabular}{|c|c|c|c|c|c|c|}
\hline & Total & CBG1 & CBG2 & CBG3 & CBG4 & $p$-value \\
\hline $\mathrm{N}(\%)$ & $1,140(100)$ & 208 (18.2) & $613(53.8)$ & $112(9.8)$ & 207 (18.2) & $<0.001$ \\
\hline Total veins & $4,378(100)$ & $819(100)$ & 2,331 (100) & 409 (100) & $819(100)$ & \\
\hline Acute PVI & 4,360 (99.6) & $812(99.1)$ & 2,324 (99.7) & 405 (99.0) & 819 (100) & 0.011 \\
\hline $28 \mathrm{~mm}$ CB \% & $812(71.2)$ & $46(22.1)$ & 468 (76.3) & $95(84.8)$ & $203(98.1)$ & $<0.001$ \\
\hline 23 mm CB \% & $63(5.5)$ & $28(13.5)$ & $35(5.7)$ & $0(0)$ & $0(0)$ & $<0.001$ \\
\hline Both balloons \% & 265 (23.2) & $134(64.4)$ & $110(17.9)$ & $17(15.2)$ & $4(1.9)$ & $<0.001$ \\
\hline LA Time min. & $80[57]$ & $145[50]$ & $80[45]$ & 80 [30] & 60 [20] & $<0.001$ \\
\hline Fluoroscopy Time min. & 26 [17] & 36 [18] & 19 [12] & 20 [11] & $14.5[7]$ & $<0.001$ \\
\hline Dose area product cGyxcm² & $1,818[2,578]$ & $4,184[3,793]$ & $1,704[2,126]$ & $1,433[1,477]$ & $1,129[1,259]$ & $<0.001$ \\
\hline
\end{tabular}

$N(\%)$ : number of patients and percentage; mean \pm standard deviation or median with interquartile range [in squared brackets] as appropriate according to the test of normal distribution. CB, cryoballoon; CBG, cryoballoon generation; LA, left atrium; PVI, pulmonary vein isolation; cGy: centigray, cm: centimeter.

Bold values are referred statistically significant.

TABLE 3 | Complications and differences in the treatment groups.

\begin{tabular}{|c|c|c|c|c|c|c|}
\hline & Total & CBG1 & CBG2 & CBG3 & CBG4 & $p$-value \\
\hline & $1,140(100)$ & $208(18.2)$ & $613(53.8)$ & $112(9.8)$ & $207(18.2)$ & \\
\hline Total major complications & $17(1.5)$ & $4(1.9)$ & $9(1.5)$ & $1(0.9)$ & $3(1.4)$ & 0.95 \\
\hline Deaths & $0(0)$ & $0(0)$ & $0(0)$ & $0(0)$ & $0(0)$ & 1.00 \\
\hline Myocardial infarction & $0(0)$ & $0(0)$ & $0(0)$ & $0(0)$ & $0(0)$ & 1.00 \\
\hline TIA/stroke & $2(0.2)$ & $0(0)$ & $2(0.3)$ & $0(0)$ & $0(0)$ & 0.63 \\
\hline Groin complications with surgery/intervention & $9(0.8)$ & $4(1.9)$ & $3(0.5)$ & $1(1.0)$ & $1(0.5)$ & 0.54 \\
\hline Arial septal defect treated by intervention & $1(0.1)$ & $0(0)$ & $1(0.1)$ & $0(0)$ & $0(0)$ & 0.83 \\
\hline Unresolved phrenic nerve palsy until discharge & $4(0.4)$ & $0(0)$ & $2(0.3)$ & $0(0)$ & $2(1.0)$ & 0.36 \\
\hline Total minor complications & $98(8.6)$ & 45 (21.6) & $38(6.2)$ & $5(4.5)$ & $10(4.8)$ & $<0.001$ \\
\hline Transient phrenic nerve palsy & $60(5.3)$ & $31(14.9)$ & $24(3.9)$ & $1(0.9)$ & $4(1.9)$ & $<0.001$ \\
\hline Total & $115(10.1)$ & 49 (23.6) & $46(7.5)$ & $6(5.4)$ & $13(6.3)$ & $<0.001$ \\
\hline
\end{tabular}

$N(\%)$ : number of patients and percentage. CBG, cryoballoon generation; TIA, transitory ischemic attack; PV, pulmonary vein.

Bold values are referred statistically significant.

\section{Outcome of CBA in Persistent AF}

After a single procedure and a median follow-up time of 22 months, atrial arrhythmia recurrences, defined as AF, atrial tachycardia, or atypical atrial flutter occurred in 520 (45.6\%) of the 1,140 patients. Kaplan-Meier estimates for arrhythmia free survival were $81.8 \%$ at 1 year, and $61.7 \%$ at 2 years of follow-up.

The Kaplan-Meier Curves in Figure 3 show the unadjusted outcome estimates according to the treatment with the firstor the next-generation cryoballoon. No difference was observed among the groups (log-rank test, $p=0.22$ ).

\section{Predictors of Recurrence}

After adjustment for baseline characteristics and treatment groups, independent risk factors for recurrence were female sex (adjusted HR 1.42, 95\% CI 1.114-1.798; $p=0.004$ ) and LA diameter determined by transthoracic echocardiography (adjusted HR 1.03, 95\% CI 1.014-1.048, $p=0.001$ ). The relative risk of recurrence increased by $3 \%$ per millimeter of the LA diameter. The use of the advanced cryoballoon generations was an independent predictor for success (adjusted HR 0.77, 95\% CI 0.605-0.976). See Table 4 for details.

\section{Durability of PVI}

From 2007 to 2020, patients with symptomatic recurrences after CBA were treated according to the guidelines, and 268 (23.5\%) of the 1,140 patients underwent a repeat ablation procedure because of symptomatic recurrences. The median time span from the initial ablation procedure to repeat ablation was 567.5 (275.5; 1191.5) days. In 96 (35.8\%) of the patients, repeat ablation was performed after first-generation CBA. The majority of patients $(172 / 268,61.3 \%)$ underwent repeat $\mathrm{AF}$ ablation by means of radiofrequency catheter ablation guided by $3 \mathrm{D}$-electroanatomical 


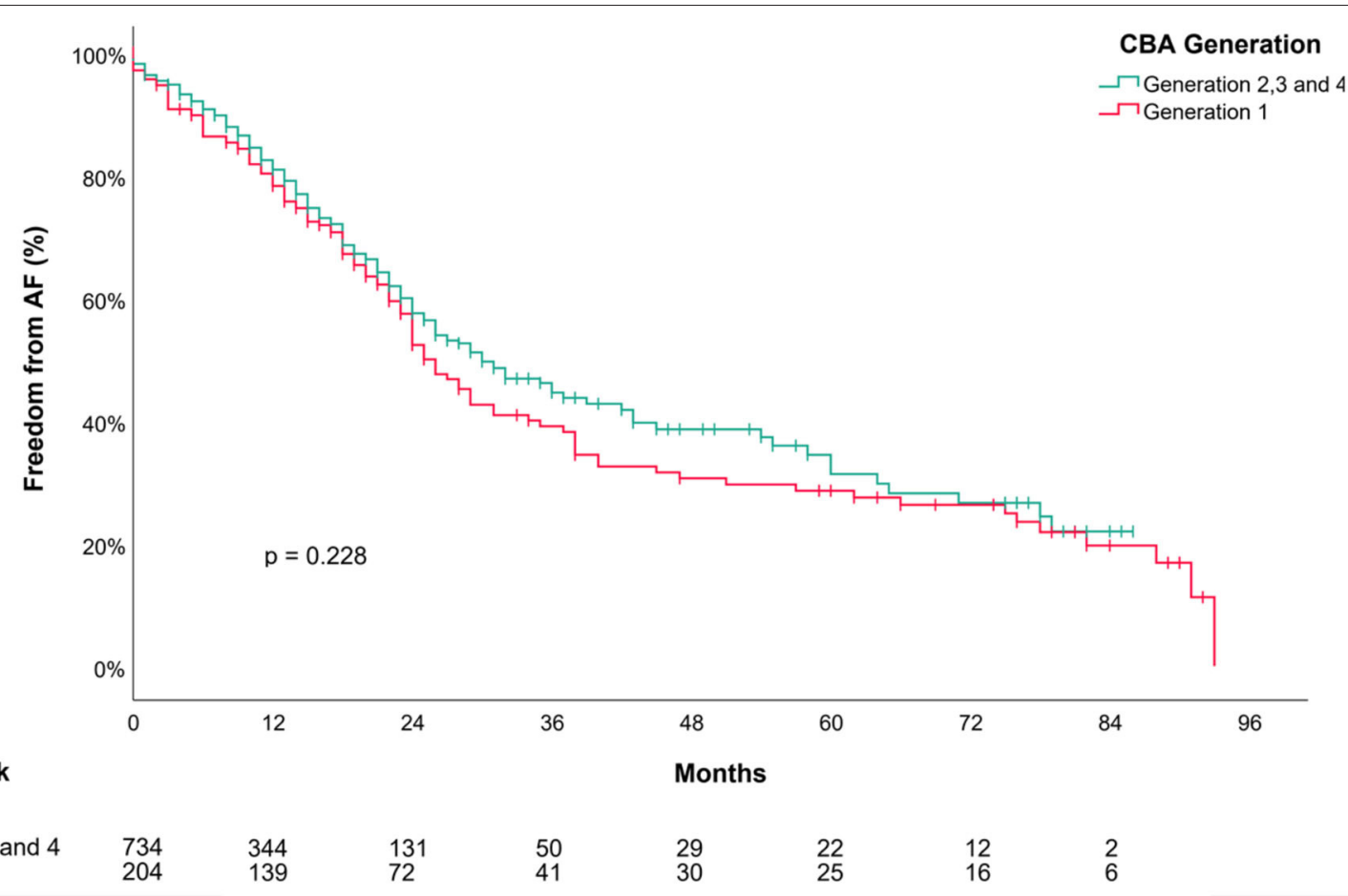

FIGURE 3 | Outcome of cryoballoon ablation in persistent AF after a single procedure. Kaplan-Meier Survival Curve depicts estimates for "Freedom from AF/AT" after a single cryoballoon ablation stratified following the first (CBG1; in red color) or an advanced cryoballoon generation (CBG2, CBG3, or CBG4; in Turquoise color) of the balloon. P-value is provided for the log-rank comparison of the curves. AF, atrial fibrillation; CBA, cryoballoon ablation.

high-density mapping after initial advanced CBA (CBG2, CBG3, or CBG4).

Of the 268 patients with a clinical indication for repeat ablation, 133 (49.6\%) demonstrated durable PVI of all veins. Patients pretreated with CBG1 demonstrated a significantly lower rate of complete PVI as compared with patients scheduled for repeat ablation after advanced CBA (18.8 vs. $66.4 \%, p<0.001)$.

The percentage of $\mathrm{PV}$ with permanent disconnection at repeat ablation, in total, was $73.5 \%$, after $\mathrm{CBG} 1$ the rate was lower (48.4\% of PVs), and after advanced CBA the rate was highest ( $88 \%$ of PVs). Details are presented in Table 5.

\section{DISCUSSION}

This is the first and largest study that evaluates PVI by means of $\mathrm{CBA}$ as the general ablation strategy in all-comers for the initial ablation procedure in symptomatic persistent AF (excluding patients with a large LA diameter of $>60 \mathrm{~mm}$ ). The main results of the study can be summarized as follows:

First, the PVI-only strategy is safe and effective in persistent AF with favorable rates of arrhythmia-free survival at 1 and 2 years after a single procedure.

Second, CBA in persistent AF creates durable PVI. At the time of the second procedure, $88 \%$ of PVs were isolated if one of the advanced CBA catheters was used.
Third, balloon technology, ablation protocols, lab management, and operator experience improved tremendously over time, and the procedure became safer, more efficacious, radiation-reduced, and faster.

Fourth, independent predictors of recurrence were female sex, LA diameter, and use of the first-generation of cryoballoon, which is no longer available.

\section{CBA as a Strategy for the Initial Procedure}

In the early days of CBA for persistent AF, the first generation of cryoballoon was used in a double balloon procedure with the rationale of wide antral ablation in persistent $\mathrm{AF}$ in our center. The initial results served as a proof of concept for CBA in persistent AF (6). The rate of acute PVI increased from $99.1 \%$ (CBG1) to $100 \%$ (CBG4), $p<0.05$. Although no difference was observed in the unadjusted log-rank comparison of the KaplanMeier plots for the outcome of first-generation vs. advanced CBA, the use of the advanced technology was an independent predictor for success after adjustment in the multivariate analysis. The high efficacy of the initial double balloon procedure with CBG1 was associated with longer left atrial dwelling times, higher amounts of radiation, more transient phrenic nerve injuries, and more pulmonary infections. Thus, the introduction of the more effective second-generation cryoballoon and its successors, with increased refrigerant flow rate, broader cooling-zone, and shorter catheter, led to better acute and chronic results despite the higher rate of comorbidities in the latter patient population. 
TABLE 4 | Predictors of outcome: uni- and multivariate analyses.

\begin{tabular}{|c|c|c|c|c|c|c|}
\hline & \multicolumn{3}{|c|}{ Univariate analysis } & \multicolumn{3}{|c|}{ Multivariate analysis } \\
\hline & HR & $95 \% \mathrm{Cl}$ & $p$-value & HR & $95 \% \mathrm{Cl}$ & $p$-value \\
\hline Age & 1.00 & $0.996-1.016$ & 0.21 & 1.00 & $0.991-1.014$ & 0.65 \\
\hline Female & 1.29 & $1.039-1.593$ & 0.02 & 1.42 & $1.114-1.798$ & 0.004 \\
\hline LA Diameter & 1.06 & $1.009-1.042$ & 0.002 & 1.03 & $1.014-1.048$ & 0.001 \\
\hline Ejection fraction & 1.00 & $0.982-1.009$ & 0.50 & & & \\
\hline AAD I/III prior to the procedure & 1.17 & $0.753-1.822$ & 0.48 & & & \\
\hline Number of electrical cardioversions & 1.00 & $0.886-1.149$ & 0.887 & & & \\
\hline Number of episodes per year & 1.00 & $0.999-1.005$ & 0.22 & & & \\
\hline Max. duration of episodes & 1.00 & $0.999-1.002$ & 0.40 & & & \\
\hline Hypertension & 0.98 & $0.779-1.235$ & 0.87 & & & \\
\hline Hypertensive heart disease & 0.96 & $0.768-1.209$ & 0.78 & & & \\
\hline Mitral regurgitation $\geq^{\circ} \|$ & 1.17 & $0.744-1.837$ & 0.49 & 1.15 & $0.719-1.829$ & 0.57 \\
\hline Coronary artery disease & 0.80 & $0.620-1.054$ & 0.12 & & & \\
\hline Prior myocardial infarction & 0.66 & $0.296-1.487$ & 0.32 & & & \\
\hline Cardiomyopathy & 1.10 & $0.775-1.547$ & 0.61 & & & \\
\hline Structural heart disease & 1.16 & $0.937-1.430$ & 0.17 & & & \\
\hline Diabetes mellitus & 0.98 & $0.455-2.124$ & 0.97 & & & \\
\hline $\mathrm{CHA}_{2} \mathrm{DS}_{2}$-VASc & 0.16 & $0.959-1.297$ & 0.16 & & & \\
\hline Body mass index & 1.00 & $0.972-1.027$ & 0.94 & & & \\
\hline Overweight (BMI > 25) & 0.87 & $0.713-1.077$ & 0.21 & & & \\
\hline Obesity (BMI > 30) & 0.97 & $0.742-1.262$ & 0.80 & & & \\
\hline Obesity ${ }^{\circ} \| / / I I(B M I ~>35)$ & 1.35 & $0.915-1.984$ & 0.13 & 1.23 & $0.812-1.868$ & 0.33 \\
\hline Common Ostium & 1.06 & $0.731-1.527$ & 0.77 & & & \\
\hline Accessory Veins & 1.12 & $0.727-1.723$ & 0.61 & & & \\
\hline Use of an advanced cryoballoon catheter ${ }^{\star}$ & 0.87 & $0.700-1.091$ & 0.23 & 0.77 & $0.605-0.976$ & 0.03 \\
\hline Only 23 mm & 0.97 & $0.670-1.411$ & 0.83 & & & \\
\hline Only 28 mm & 0.56 & $0.763-1.159$ & 0.56 & & & \\
\hline $23+28 \mathrm{~mm}$ & 1.08 & $0.869-1.343$ & 0.49 & & & \\
\hline
\end{tabular}

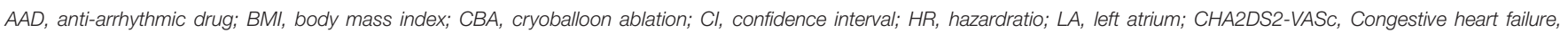
Hypertension, Age $\geq 75$ years (doubled), Diabetes mellitus, prior Stroke or TIA or thromboembolism (doubled), Vascular disease, Age 65 to 74 years, Sex category.

${ }^{*} \mathrm{CBG} 2, \mathrm{CBG} 3$, or CBG4.

Bold values are referred statistically significant.

The implementation of smart ablation protocols with dosing strategies according to the individual time to electrical isolation of the PV contributed to reduced application times, which turns into shorter LA dwelling times without compromising the efficacy.

\section{Safety of CBA}

Overall, CBA in the initial ablation procedure for persistent AF is safe. During more than 10 years of experience and 1,140 patients treated, no death was associated with the procedure and no atrio-esophageal fistula was occurred. The very low thromboembolic cerebrovascular complication rate could be explained by the lesions characteristics of cryoenergy in cardiac tissue, and minimal endocardial surface disruption (15). In addition, the rate of pericardial tamponade was also extremely low $(1 / 1,140[0.09 \%])$ and might be attributed to the overthe-wire balloon platform, single-transseptal access approach, cryothermal energy source itself, experience of the operators, and use of echocardiographic (transesophageal echocardiography,
TEE, or intracardiac echocardiography, ICE) guidance in all the cases. The observed rate of persistent phrenic nerve palsy was very low as compared with the literature (16), and the rate of transient phrenic nerve injury decreased significantly over time. Four main determinants were identified for the decline in transient PNP: experience of the operators, additional visualization of the diaphragmatic motion by ICE, abolition of the routine double balloon strategy and of the routine bonus application, and reduction in application times. Of note, neither the implementation of uninterrupted or shortly interrupted oral anticoagulation nor the implementation of propofol/morphine analgo-sedation had an impact on the safety profile of the procedure.

\section{Radiation Exposure}

Radiation dose decreased significantly over time. From 2007 to 2020, all the procedures were performed on the same catheter lab x-ray system (Philips Allura cardiovascular Xray system; Philips, Eindhoven, Netherlands) with presets 
TABLE 5 | Pulmonary vein electrical reconnection at repeat ablation.

\begin{tabular}{|c|c|c|c|}
\hline $\begin{array}{l}\text { Number of PV with } \\
\text { reconnection }\end{array}$ & $\begin{array}{l}\text { Total number of patients with } \\
\text { repeat ablation } N=268\end{array}$ & $\begin{array}{c}\text { Number of patients with repeat ablation } \\
\text { after first generation CBA } N=96\end{array}$ & $\begin{array}{l}\text { Number of patients with repeat } \\
\text { ablation after advanced } C^{*} A^{*} N=172\end{array}$ \\
\hline 0 & $133(49.6)$ & $18(18.8)$ & $115(66.4)$ \\
\hline 1 & $60(22.4)$ & $18(18.8)$ & $42(24.3)$ \\
\hline 2 & $36(13.4)$ & $25(26.0)$ & $11(6.4)$ \\
\hline 3 & $14(5.2)$ & 13 (13.5) & $1(0.57)$ \\
\hline 4 & $24(9.0)$ & $21(21.9)$ & $3(1.7)$ \\
\hline 5 & $1(0.4)$ & $1(1.0 \%)$ & $0(0)$ \\
\hline PV & & $\%$ of PV with durable isolation & \\
\hline all PV & 73.5 & 48.4 & 88.0 \\
\hline LSPV & 73.2 & 47.3 & 88.1 \\
\hline LIPV & 78.1 & 53.3 & 92.5 \\
\hline RIPV & 73.6 & 45.7 & 89.6 \\
\hline RSPV & 70.8 & 49.5 & 82.9 \\
\hline RMPV & 45.5 & 42.9 & 50.0 \\
\hline LCPV & 55.6 & 0.0 & 83.3 \\
\hline
\end{tabular}

Numbers with percentages.

$P V$, pulmonary vein; LCPV, left common PV; LIPV, left inferior PV; LSPV, left superior PV; RIPV, right inferior PV; RMPV, right middle PV; RSPV, right superior PV. *CBG2, CBG3, or CBG4.

for electrophysiological procedures. Dose reduction might be attributed to the practical implementation of the "As Low As Reasonably Achievable" (ALARA) principle in our center with shorter cine sequence acquisition, better collimation, reduced frame rates, and less angulation. In addition, the shorter LA dwelling times, improved steerable sheath, long-term learning curve of the operators, implementation of ICE in the lab, and significant lower BMI of the patients might have led to the reduction in radiation exposure.

\section{Predictors of Recurrence}

Left atrial diameter was a strong independent predictor of recurrence. This result proves the observation of Reissmann et al. (17) and Akkaya et al. (18) on a larger population.

Female sex was an independent predictor for atrial arrhythmia recurrence in our study. Women with AF are older and have a higher prevalence of comorbidities compared with males (19). The relative risk of recurrences in women was $42 \%$ higher as compared with males ( $p=0.004$ in the multivariate analysis). In the 1STOP trial, with mainly patients with paroxysmal AF, female sex has also been reported to be an independent predictor for recurrence after CBA (20). Neither Reissmann nor Akkaya has found sex-related differences in CBA for persistent $\operatorname{AF}(17,18)$. However, both of the studies were smaller, with 135 and 102 patients, respectively. Hypothetically, sex-related differences in the presence of fibrotic atrial myopathy or additional extra-PV trigger sites may explain our observations.

\section{Outcome and Durability of PVI}

In the meta-analysis of Omran, freedom from AF following CBA in persistent AF was $68.9 \%$ after a mean FU of $16.7 \pm 3$ months (8). In the CRYO4PERSISTENT and the STOP Persistent AF trials, a 12-month success rate of 61 and 55\%, respectively, was reported $(7,21)$. In our study, recurrences were observed in $45.6 \%$ of the patients, and the Kaplan-Meier estimates for arrhythmia-free survival was $81.8 \%$ at 1 year, and $61.7 \%$ at 2 years of follow-up. At 1-year follow-up, Ciconte et al. demonstrated that $60 \%$ of patients had stable sinus rhythm following persistent AF ablation with the novel cryoballoon (22). Differences in outcomes among centers might be influenced by differences in baseline characteristics, follow-up methods, operator experience, and definition of endpoints.

The favorable outcome with respect to arrhythmia-free survival at 1 - and 2-years in our study might be associated with the durability of PVI following CBA in persistent AF. CBA is supposed to create less operator-dependent, reproducible, and comparable outcome results among centers, as reported by Providencia et al. (23). In paroxysmal AF, a rate of $1.4 \pm 1.1$ reconnected PV at repeat ablation has been demonstrated (24). Data on the rate of complete PVI in patients with persistent AF following initial sole cryoballoon PVI are scarce. All PVs were durably isolated in 66 of 81 (81.5\%) patients with persistent AF following PVI plus posterior wall isolation using the cryoballoon (21). This manuscript includes the largest report on the durability of PVI following index CBA in persistent AF. Complete electrical PVI was present in $50 \%$ of the patients at repeat ablation. In the initial procedure, most of the patients were treated with one of the advanced, more effective cryoballoon catheters. Their higher efficacy was also demonstrated in our study by the significant increase in the durability of PVI from $18.8 \%$ after initial CBG1 ablation to $66.4 \%$ of the patients after initial advanced CBA. The fact that $88 \%$ of PVs in patients with symptomatic recurrences are still durably isolated after initial advanced CBA underscores the need for ablation strategies beyond PVI at the repeat procedure. The benefit of novel ultra-high density mapping systems in guiding ablation strategies in patients with recurrences and durable isolated PV following CBA has been reported recently (25). 


\section{Limitations}

This was a single-center observational study with inherent limitations accompanying this type of study design. The results were based on clinical visits and evaluation of symptoms, ECG, and Holter monitoring (1-7 days) during routine follow-up. Therefore, asymptomatic recurrence might have been missed. On the other hand, the recurrence rate might be overestimated, as typical symptoms in patients with paroxysmal AF were counted as recurrence if ECG documentation was difficult. No systematic, continuous monitoring with implantable devices was available.

In this trial, available cryoballoon ablation catheters (Medtronic Inc., Minneapolis, MN, United States) were used. It is unclear if these results are reproducible by novel cryoballoon systems.

\section{CONCLUSION}

Pulmonary vein isolation in symptomatic persistent AF is a reasonable strategy in the initial ablation procedure for rhythm control. Moreover, CBA in this setting provides a safe and effective technique with low radiation exposure, short left atrial dwelling times, favorable outcomes, and high rates of durably isolated PVs after a single procedure. Our results underscore the need for ablation strategies beyond PVI for repeat AF ablation procedures, because PV reconnection is no longer a common finding with the currently available cryoballoon technology.

\section{DATA AVAILABILITY STATEMENT}

The datasets presented in this article are not readily available because the database was kept on the server of the Heart-Center Munich-Bogenhausen, Munich, Germany. The authors confirm that the data supporting the findings of this study are available within the article and/or its supplementary materials. Due to the nature of this research, participants of this study did not agree for their data to be shared publicly, so supporting raw data is not available. Requests to access the datasets should be directed to florian.straube@muenchen-klinik.de.

\section{REFERENCES}

1. Hindricks G, Potpara T, Dagres N, Arbelo E, Bax JJ, Blomstrom-Lundqvist C, et al. 2020 ESC Guidelines for the diagnosis and management of atrial fibrillation developed in collaboration with the European Association for Cardio-Thoracic Surgery (EACTS). Eur Heart J. (2021) 42:373498. doi: 10.1093/eurheartj/ehaa612

2. Calkins H, Hindricks G, Cappato R, Kim YH, Saad EB, Aguinaga L, et al. 2017 HRS/EHRA/ECAS/APHRS/SOLAECE expert consensus statement on catheter and surgical ablation of atrial fibrillation. Heart Rhythm. (2017) 14:e275-444. doi: 10.1016/j.hrthm.2017.05.012

3. Kuniss M, Pavlovic N, Velagic V, Hermida JS, Healey S, Arena G, et al. Cryoballoon ablation vs. antiarrhythmic drugs: first-line therapy for patients with paroxysmal atrial fibrillation. Europace. (2021) 23:103341. doi: 10.1093/europace/euab029

4. Wazni OM, Dandamudi G, Sood N, Hoyt R, Tyler J, Durrani S, et al. Cryoballoon ablation as initial therapy for atrial fibrillation. $N$ Engl J Med. (2021) 384:316-24. doi: 10.1056/NEJMoa2029554

\section{ETHICS STATEMENT}

The studies involving human participants were reviewed and approved by Bavarian State Camber of Physicians. The patients/participants provided their written informed consent to participate in this study.

\section{AUTHOR CONTRIBUTIONS}

FS analyzed the data, interpreted the results, and drafted the manuscript and figures. JP analyzed the data, was responsible for the statistics, drafted tables and figures, and interpreted the results. This study contains data from the doctoral thesis of $\mathrm{AK}$, who contribute data acquisition, follow-up, and data analysis and interpretation. UD, EH, and FS designed the study protocol. FS, UD, LR, BB, SH, CT, UE, and MW contributed to patient recruitment and data acquisition. $\mathrm{BB}$ was responsible for data management. $\mathrm{EH}$ conducted the study as the head of the department, substantially contributed to the conception of the study, patient recruitment, data acquisition, and interpretation of the results. All authors substantially contributed to the study, critically revised the manuscript for important intellectual content, approved the manuscript, and agreed to be accountable for all aspects of the study.

\section{FUNDING}

AK and JP received scholarships from the independent nonprofit Zenner Foundation, Munich, Germany.

\section{ACKNOWLEDGMENTS}

A special thanks goes to the assistant personnel of our EP lab who supported our study every day and provided a very comfortable setting not only for the physicians, but especially for the patients. We thank Mrs. June Tomelden and Mrs. Sophie Rump, the local study nurses, for the help with data management.

5. Straube F, Dorwarth U, Ammar-Busch S, Peter T, Noelker G, Massa T, et al. First-line catheter ablation of paroxysmal atrial fibrillation: outcome of radiofrequency vs. cryoballoon pulmonary vein isolation. Europace. (2016) 18:368-75. doi: 10.1093/europace/euv271

6. Schmidt M, Dorwarth U, Straube F, Wankerl M, Krieg J, Leber AW, et al. A novel double cryoballoon strategy in persistent atrial fibrillation: a pilot study. Clin Res Cardiol. (2012) 101:777-85. doi: 10.1007/s00392-012-0456-y

7. Boveda S, Metzner A, Nguyen DQ, Chun KRJ, Goehl K, Noelker G, et al. Single-procedure outcomes and quality-of-life improvement 12 months post-cryoballoon ablation in persistent atrial fibrillation: results from the multicenter CRYO4PERSISTENT AF trial. JACC Clin Electrophysiol. (2018) 4:1440-7. doi: 10.1016/j.jacep.2018.07.007

8. Omran H, Gutleben KJ, Molatta S, Fischbach T, Wellmann B, Horstkotte $\mathrm{D}$, et al. Second generation cryoballoon ablation for persistent atrial fibrillation: an updated meta-analysis. Clin Res Cardiol. (2018) 107:18292. doi: 10.1007/s00392-017-1171-5

9. Dorwarth U, Schmidt M, Wankerl M, Krieg J, Straube F, Hoffmann E. Pulmonary vein electrophysiology during cryoballoon ablation as a 
predictor for procedural success. J Interv Card Electrophysiol. (2011) 32:20511. doi: $10.1007 / \mathrm{s} 10840-011-9585-\mathrm{x}$

10. Hartl S, Dorwarth U, Bunz B, Wankerl M, Ebersberger U, Hoffmann E, et al. Lessons from individualized cryoballoon sizing. Is there a role for the small balloon? J Cardiol. (2017) 70:374-81. doi: 10.1016/j.jjcc.2016. 12.016

11. Straube F, Dorwarth U, Hartl S, Bunz B, Wankerl M, Ebersberger U, et al. Outcome of paroxysmal atrial fibrillation ablation with the cryoballoon using two different application times: the 4- versus 3-min protocol. J Interv Card Electrophysiol. (2016) 45:169-77. doi: 10.1007/s10840-015-0084-3

12. Straube F, Dorwarth U, Pongratz J, Bruck B, Wankerl M, Hartl S, et al. The fourth cryoballoon generation with a shorter tip to facilitate real-time pulmonary vein potential recording: Feasibility and safety results. J Cardiovasc Electrophysiol. (2019) 30:918-25. doi: 10.1111/jce.13927

13. Straube F, Dorwarth U, Schmidt M, Wankerl M, Ebersberger U, Hoffmann E. Comparison of the first and second cryoballoon: high-volume singlecenter safety and efficacy analysis. Circ Arrhythm Electrophysiol. (2014) 7:2939. doi: 10.1161/CIRCEP.113.000899

14. Straube F, Hartl S, Dorwarth U, Wankerl M, Bunz B, Ebersberger U, et al. Cryoballoon ablation for persistent atrial fibrillation - Large single-center experience. J Cardiol. (2016) 68:492-7. doi: 10.1016/j.jjcc.2016.02.007

15. Khairy P, Chauvet P, Lehmann J, Lambert J, Macle L, Tanguay $\mathrm{JF}$, et al. Lower incidence of thrombus formation with cryoenergy versus radiofrequency catheter ablation. Circulation. (2003) 107:2045-50. doi: 10.1161/01.CIR.0000058706.82623.A1

16. Tohoku S, Chen S, Last J, Bordignon S, Bologna F, Trolese L, et al. Phrenic nerve injury in atrial fibrillation ablation using balloon catheters: Incidence, characteristics, and clinical recovery course. J Cardiovasc Electrophysiol. (2020) 31:1932-41. doi: 10.1111/jce.14567

17. Reissmann B, Plenge T, Heeger CH, Schluter M, Wohlmuth P, Fink T, et al. Predictors of freedom from atrial arrhythmia recurrence after cryoballoon ablation for persistent atrial fibrillation: A multicenter study. J Cardiovasc Electrophysiol. (2019) 30:1436-42. doi: 10.1111/jce.14023

18. Akkaya E, Berkowitsch A, Zaltsberg S, Greiss H, Hamm CW, Sperzel J, et al. Second-generation cryoballoon ablation for treatment of persistent atrial fibrillation: Three-year outcome and predictors of recurrence after a single procedure. J Cardiovasc Electrophysiol. (2018) 29:38-45. doi: 10.1111/jce.13372

19. Linde C, Bongiorni MG, Birgersdotter-Green U, Curtis AB, Deisenhofer I, Furokawa T, et al. Sex differences in cardiac arrhythmia: a consensus document of the European Heart Rhythm Association, endorsed by the Heart Rhythm Society and Asia Pacific Heart Rhythm Society. Europace. (2018) 20:1565. doi: 10.1093/europace/euy067

20. Ricciardi D, Arena G, Verlato R, Iacopino S, Pieragnoli P, Molon G, et al. Sex effect on efficacy of pulmonary vein cryoablation in patients with atrial fibrillation: data from the multicenter real-world 1STOP project. J Interv Card Electrophysiol. (2019) 56:9-18. doi: 10.1007/s10840-019-00601-3

21. Aryana A, Di Biase L, Pujara DK, Baker JH, Espinosa MA, de Asmundis C, et al. Long-term durability of posterior wall isolation using the cryoballoon in patients with persistent atrial fibrillation: a multicenter analysis of repeat catheter ablations. J Interv Card Electrophysiol. (2020) 62:1619. doi: 10.1007/s10840-020-00887-8

22. Ciconte G, Ottaviano L, de Asmundis C, Baltogiannis G, Conte G, Sieira J, et al. Pulmonary vein isolation as index procedure for persistent atrial fibrillation: One-year clinical outcome after ablation using the second-generation cryoballoon. Heart Rhythm. (2015) 12:60-6. doi: 10.1016/j.hrthm.2014.09.063

23. Providencia R, Defaye P, Lambiase PD, Pavin D, Cebron JP, Halimi F, et al. Results from a multicentre comparison of cryoballoon vs. radiofrequency ablation for paroxysmal atrial fibrillation: is cryoablation more reproducible? Europace. (2017) 19:48-57. doi: 10.1093/europace/euw080

24. Kuck KH, Albenque JP, Chun KJ, Furnkranz A, Busch M, Elvan A, et al. Repeat ablation for atrial fibrillation recurrence post cryoballoon or radiofrequency ablation in the FIRE and ICE trial. Circ Arrhythm Electrophysiol. (2019) 12:e007247. doi: 10.1161/CIRCEP.119.007247

25. Straube F, Dorwarth U, Hartl S, Brueck B, Pongratz J, Kosmalla A, et al. Benefit of ultra-high-density mapping-guided radiofrequency reablation in pulmonary vein isolation non-responders after initial cryoballoon procedure. Europace. (2020) 22:906-15. doi: 10.1093/europace/euaa055

Conflict of Interest: FS received honoraria for lectures from Medtronic and Bristol-Myers-Squibb outside the submitted study and educational support from Pfizer. UD reports honoraria for lectures from Medtronic Inc., outside the submitted study. SH participates in the EP fellowship from Boston Scientific, received educational support from Biotronik, Daiichi Sankyo, and honoraria for lectures from Bristol Myers Squibb outside the submitted study. EH was head of the department; the department received compensation for participation in clinical research trials outside the submitted study from Abbott, Bayer, Biotronik, Boehringer Ingelheim, Edwards, Elixier, Medtronic, and Stentys.

The remaining authors declare that the research was conducted in the absence of any commercial or financial relationships that could be construed as a potential conflict of interest.

Publisher's Note: All claims expressed in this article are solely those of the authors and do not necessarily represent those of their affiliated organizations, or those of the publisher, the editors and the reviewers. Any product that may be evaluated in this article, or claim that may be made by its manufacturer, is not guaranteed or endorsed by the publisher.

Copyright (c) 2021 Straube, Pongratz, Kosmalla, Brueck, Riess, Hartl, Tesche, Ebersberger, Wankerl, Dorwarth and Hoffmann. This is an open-access article distributed under the terms of the Creative Commons Attribution License (CC BY). The use, distribution or reproduction in other forums is permitted, provided the original author(s) and the copyright owner(s) are credited and that the original publication in this journal is cited, in accordance with accepted academic practice. No use, distribution or reproduction is permitted which does not comply with these terms. 